\title{
Preparation Chitosan Lactate-Hyaluronate Sponges with Unidirectional Porous Structure and Their Potential Use as Wound Dressings
}

\author{
Chen Lai
}

\begin{abstract}
Sponges were prepared from lactic acid-grafted chitosan (LCH) and hyaluronate (HA) to develop potential materials for wound dressing. Sponges with different microstructures can be obtained in a vertical temperature-controlled device and in an aluminum vessel. The morphology and permeability properties, including porosity, water permeability, gas permeability, water adsorption and in vitro biodegradation of two types of sponges were compared in detail. Sponges with microtubule-oriented structure can be obtained in a vertical temperature-controlled device with TBA (tertiary butyl alcohol). These sponges exhibited an applicable water permeation rate of $577 \mathrm{~g} \cdot \mathrm{m}^{-2} \cdot \mathrm{day}^{-1}$ and higher air permeability tensor of $3 \times 10^{-12}$. The sponges with random micropores can be prepared in an aluminum vessel. These sponges exhibited a water permeation rate and an air permeability tensor of $452 \mathrm{~g} \cdot \mathrm{m}^{-2} \cdot \mathrm{day}^{-1}$ and $1.8 \times 10^{-14}$ $\mathrm{g} \cdot \mathrm{m}^{-2} \cdot \mathrm{day}^{-1}$, respectively. These results also indicated that TBA greatly affected the microstructure of the resulting sponges. A parallel orientation of the pore structure resulted in higher porosity and higher water adsorption capacity than random pores. After lactic acid was grafted, the crystallinity of chitosan decreased and water solubility increased, causing rapid lysozymatic degradation of chitosan lactate-HA sponges. The resulting sponges exhibited high water content, gas permeability and appropriate water permeation; such sponges could easily be applied and removed. These properties indicated that these materials are highly applicable as wound dressing.
\end{abstract}

Index Terms - Chitosan lactate, hyaluronate, sponge.

\section{INTRODUCTION}

$\mathrm{CH}$ is a modified carbohydrate polymer derived from the chitin component of the shells of crustacean such as crab, shrimp and cuttlefish. It is a linear copolymer of glucosamine and $\mathrm{N}$-acetylglucosamine units linked by $\beta$ - 1 4-bonds. Due to its biocompatibility, low toxicity and degradation by enzymes such as chitosanase and lysozyme [1], $\mathrm{CH}$ has been examined to be used in a wide variety of biomedical applications, including tissue engineering [2], drug carrier [3] and wound healing [4]. In order to increase its dissolubility in water, $\mathrm{CH}$ is modified by chemical grafting [5], enzymatic modification [6] or reacted with acid to form soluble $\mathrm{CH}$-salt [7]. Among these chitosans derivatives, chitosan lactate (LCH) exhibits great biocompatibility, antibacteria and low toxicity. The ranking of cytotoxicity among these derivatives is chitosan hydrochloride > chitosan glutamate > chitosan lactate [8]. Some researchers' studies suggest that LCH is a

Manuscript received August 12, 2013; revised December 2, 2013.

C. Lai is with the Shenzhen key laboratory of human tissue regeneration and repair, Shenzhen institute, Peking University, Shenzhen 518057, China (e-mail: laichen1110@msn.com). safe and efficient gene carrier [9]. It also has been used to develop CPC (calcium phosphate cement) composites with higher strength and increased strain before failure [10]. Various products made from $\mathrm{CH}$ are being developed for use as wound dressings. In a comparison of several types of dressings, the LCH dressing performed best, providing a very soft, flexible, pliable bioadhesive dressing [11]. HA is one of the glycosaminoglycan components in natural extracellar matrices. It is a linear, unbranched polymer which is composed of a repeating disaccharide that consists of $\mathrm{N}$-acetyl-D-glucosamine and D-glucuronic acid linked by a $\beta$ 1-4 glycosidic bond. HA was first isolated as an acid, but at physiological conditions it behaved like a salt (sodium hyaluronate). HA plays a significant role not only in wound healing [11] but in skin for cosmetic purposes [12].

It is estimated that approximately two million people in the United States suffer from some type of chronic wound. And there are about 400 million patients need skin transplant every year in China. As we mentioned above, $\mathrm{CH}$ and HA have shown excellent potential for skin wound dressing. However, the direct mixture of them may not be an ideal material for wound dressing. $\mathrm{CH}$ is a weak base, a positively charged polyelectrolyte in acidic condition and becomes insoluble at approximately $\mathrm{pH} 6.5$ while $\mathrm{HA}$ has a negative charge at $\mathrm{pH}$ 7.4. Precipitation occurs after mixing them directly at higher concentration due to their opposite charges. Very thin film can be formed only at lower concentration. Polysaccharide nanofilms with $0.03 \sim 2.4 \mu \mathrm{m}$ in thickness were formed by dipping sliders in $\mathrm{CH}$ and $\mathrm{HA}$ acid alternately [13], [14]. It is an ideal multilayer film for oral ulcer or blood vessel reparation [15], [16]. However, it is too thin to be the dermis substitute because human skin dermis is in the range of $0.5 \sim 2 \mu \mathrm{m}$. At this aspect, $\mathrm{LCH}$ is more favorable than $\mathrm{CH}$ because it can form malleable and fluid matrix with HA [17]. CH and many of its derivatives are semi-crystal polymer and their membrane often have lower gas permeability in original state [18]. Based on the mechanism of capillarity, we can conceive the idea of capillary wound dressings which can absorb the wound exudates by capillary action but impermeable to water and bacteria. This kind of structure would maintain a moist wound surface and allow gas exchange.

Porous film or sponge with unidirectional pore structure may act as capillary action wound dressing. The unidirectional pore structure could be formed by freezing and lyophilizing solution in suitable molds. By varying the thermal gradients and freezing rate, ice crystal can solidify unidirectionally from the solution during the freezing process. Ice removal by lyophilization generates a unidirectional porous structure [19], [20]. TBA is a widely used co-solvent 
and acts as a mass-transfer accelerator in lyophilization processes. It is miscible with water in any proportion and could be removed rapidly and completely by freeze-drying because of its high vapor pressure and high melting point. Moreover, the toxicity of it is low [21]. The research of Kasraian revealed that TBA can have great effect on the crystal habit of ice [22]. By controlling the morphologies of ice, the pore structure of sponges or film can be tuned finely.

The aim of the present work was to develop freezing-dry process to manufacture $\mathrm{LCH}^{-} \mathrm{HA}$ sponges with unidirectional porous structure and adjustable pore size for medical application. Due to the outstanding moisture keeping ability of HA and the biodegradablity of $\mathrm{LCH}$, this wound dressing would easy to apply and remove without causing trauma either to the granulation tissue or the new epithelium. Sponges made by different manufacturing techniques were compared in terms of their porous morphologies, porosity, water vapor permeation, gas permeation in wet state, water uptake capacity and in vitro biodegradation. Controlling unidirectional porous morphology is the key issues to be addressed in the experiments.

\section{EXPERIMENTAL}

\section{A. Materials}

$\mathrm{CH}(\mathrm{Mw}=389,000$ and $92 \%$ deacetylated $)$, lactic acid aqueous solution $[85 \%(\mathrm{w} / \mathrm{w})]$, sodium hyaluronate $(\mathrm{Mw}=$ 1.5 DMa), chicken egg lysozyme (20000 U/mg, Fluka, USA), and TBA were purchased from Sigma Aldrich Co. (Milwaukee, USA) and used without further purification.

\section{B. Preparation of $\mathrm{CH}$ Lactate}

$\mathrm{CH}(0.8 \mathrm{~g})$ and lactate acid aqueous solution $(0.5 \mathrm{~mL})$ were dispersed in $40 \mathrm{ml}$ of deionized water. The mixture was stirred overnight at room temperature, and a clear light yellow viscous aqueous solution was obtained. The resulting solution was frozen in a refrigerator at $-78^{\circ} \mathrm{C}$ for $24 \mathrm{~h}$. The frozen hydrogel was lyophilized in a freeze-dryer for $48 \mathrm{~h}$. The light yellow and fluffy powders of LCH were fabricated.

\section{Preparation of LCH-HA Sponges}

Samples prepared in the device with vertical thermal gradient. The desired amounts of sodium HA and LCH were dissolved in distilled water, forming aqueous solutions with concentrations of 0.5 and $30 \mathrm{~g} / \mathrm{L}$, respectively. The mixture was stirred for $12 \mathrm{~h}$. Afterwards, 5\%, 12\%, 20\%, 30\%, and $40 \%(\mathrm{w} / \mathrm{w})$ TBA were added to the resulting solution and stirred for $30 \mathrm{~min}$. The resulting solution was then poured into the freezing device (Fig. 1). To further assess the effect of TBA on the material, we performed analogous experiments without TBA in the same freezing device.

The device consisted of two parts: a solution container and a coolant reservoir. The massive copper block in the coolant reservoir was used as a thermal conductor and continuously cooled by liquid nitrogen $\left(-196^{\circ} \mathrm{C}\right)$. The side of the solution container was composed of polytetrafluoroethylene and surrounded by polyurethane, which are excellent heat-conserving materials. The top layer of the solution container was exposed to the atmosphere at room temperature. The device yielded an approximately vertical thermal gradient, leading to the vertical growth of ice crystals. The samples were frozen in liquid nitrogen $\left(-196^{\circ} \mathrm{C}\right)$, stored in a freezer at $-78^{\circ} \mathrm{C}$ for $24 \mathrm{~h}$ and lyophilized in freeze-dryer (Christ Loc-1m, Germany) for $48 \mathrm{~h}$.

In the present study, $0.5 \mathrm{~g} / \mathrm{L}$ of sodium hyaluronate aqueous solution was mixed with $30 \mathrm{~g} / \mathrm{L}$ of $\mathrm{LCH}$ aqueous solution and stirred overnight. The mixture was transferred to a sealed aluminum vessel. The vessel was then immersed in liquid nitrogen and stored in a freezer at $-78^{\circ} \mathrm{C}$ for $24 \mathrm{~h}$. The mixture was subsequently lyophilized in a freeze-dryer (Christ Loc-1m, Germany) for $48 \mathrm{~h}$.

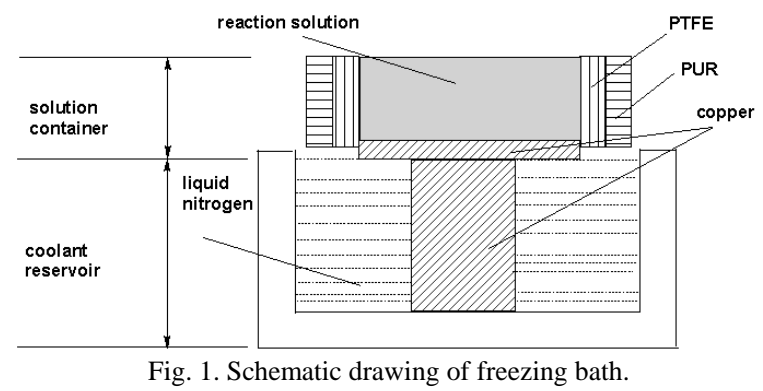

\section{Characterization of Morphology and PBS Solution Adsorption}

Surface and pore morphology were investigated by scanning electron microscopy (30XLFEG, Philips). The samples were immersed in liquid nitrogen, ground to small pieces and sputtered with a Au layer.

Each sponge was soaked in PBS buffer and allowed to reach equilibrium at $37{ }^{\circ} \mathrm{C}$ for $3 \mathrm{~d}$. The sponges were removed from water and hung for $1 \mathrm{~min}$ until no water dripped. The sponges were then weighed. The PBS solution uptake $\left(S_{P}\right)$ of the sponge was calculated using the following equation:

$$
S_{p}=\frac{W_{W}}{W_{D}}
$$

where $W w$ is the weight of wet sponge and $W_{D}$ is the weight of dry sponge.

\section{E. Water Vapor Permeability (WVTR) and Air Permeability}

WVTR was calculated using the following equation:

$$
W V T R=\left(\frac{P}{L}\right) \bullet\left(P_{W}\right) \bullet \Delta(R H)
$$

where $P$ is the water vapour permeability, which is defined as the amount of water that passes through a unit membrane thickness per unit surface area per unit time by unit vapor pressure difference, and $\triangle R H$ is the relative humidity difference across the sponge. $P_{W}$ is the saturated water vapor pressure at the experimental temperature. In the experiments, WVTR was calculated by dividing the slope of water loss versus time curve by the transmission area. WVTR was determined according to the ASTM method E96-90 at $30{ }^{\circ} \mathrm{C}$ with $31 \%$ relative humidity gradient across the dressing. The area of the dressing was $1.38 \times 10^{-3} \mathrm{~m}^{2}$. Each experiment was performed in triplicate. 
Air permeation rates were determined according to the ISO 4638 and $\mathrm{GB} / \mathrm{T} 10655-2003$ at $30{ }^{\circ} \mathrm{C}$ with a $97.1 \%$ relative humidity gradient across the dressing. These experiments were performed in triplicate. Air transmission rate $(u)$ was calculated according to Darcy's law, which describes the flow of a fluid through a porous medium, by using the following equation:

$$
u=\frac{K \bullet \Delta P}{\eta \delta}
$$

\section{F. In Vitro Degradation}

The biodegradation of the sponge was investigated in vitro by incubating the sponge in $0.15 \mathrm{M} \mathrm{NaCl}$ (pH 5) with 1 $\mathrm{mg} / \mathrm{mL}$ of lysozyme in an incubation dish and maintained at $37{ }^{\circ} \mathrm{C}$. The degradable ratio $(D)$ was calculated using the following equation:

$$
D=\frac{W_{0}-W_{t}}{W_{0}} \times 100
$$

where $W_{0}$ is the original weight of the sponge and $W_{t}$ is the weight at different degradation times. In vitro lysozyme degradation experiments were performed five times.

\section{G. Porosity Estimation}

The porosity of the $\mathrm{LCH}$ sponge cannot be measured using a mercury intrusion porosimeter because of the comprisable texture of the sponge. Therefore, the porosity $(\varepsilon)$ of the sample was calculated using the following equation:

$$
\varepsilon=\frac{V_{P}}{V_{t}}=\left(1-\frac{\rho_{1}}{\rho_{2}}\right) \times 100
$$

where $V_{P}$ is the pore volume, $V_{t}$ is the volume of the sample, including the pore volume and the sponge volume, $P_{1}$ is the density of the initial material and $P_{2}$ is the density of the swollen materials, which were immersed in ethanol.

\section{RESULTS AND DISCUSSION}

\section{A. Sponge Morphology}

Pore morphology can be controlled by using different processing methods. The morphology of LCH-HA sponges is shown in Fig. 2 and Fig. 3. A microtubular shape and oriented porous arrangement can be achieved in sponges prepared in the controlled device (Fig. 2 c) and Fig. 2 d) ). For the samples frozen in an aluminum vessel, random micropores with numerous macrovoids can be observed (Fig. 2 a) ]. These totally different structures are possibly formed as a result of the differences in ice nucleation and growth conditions. Under freezing conditions, the solution separates into a pure ice phase and a solvent phase. After the ice sublimated during freeze drying, the porous structure resembled the crystal morphology of ice. Very small crystals were formed during rapid freezing in an aluminum vessel with high thermal conductivity. The sealed vessel with uniform heat transfer can potentially induce the homogeneous nucleation of ice crystals, and these crystals grew in different directions. In the case of the aluminum vessel, heat was transferred from the surroundings to the sample via the metal wall of the vessel, thereby causing ice nucleation on the wall. As a result, ice crystal growth was oriented inward. Freezing, particularly at very high rates, may promote air sealing, in which air is instantly enveloped in samples when ice is completely formed.

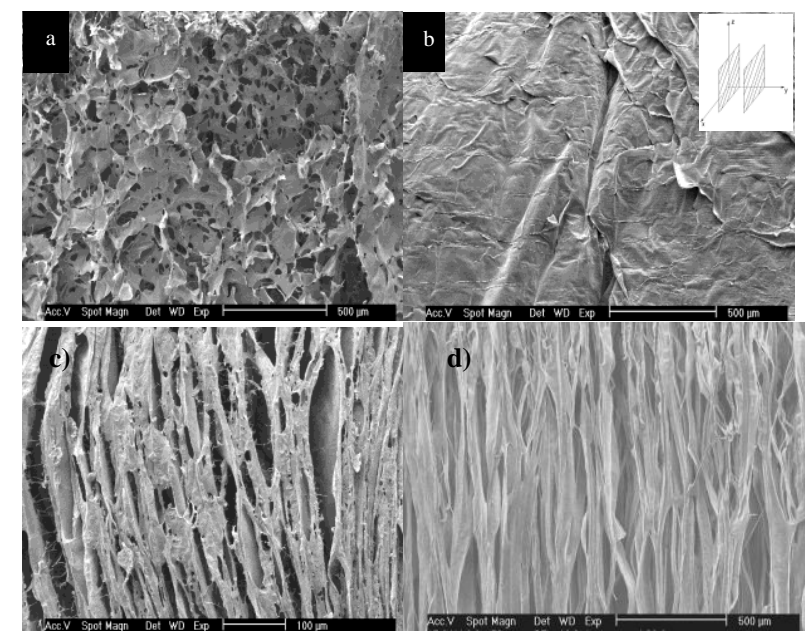

Fig. 2. SEM images of the LCH-HA sponges frozen under various condition.

The cross sections is parallel to the temperature gradient. Fig. 2 a). The sample was prepared in sealed aluminum vessel by immersion in liquid nitrogen; Fig. 2 b), Fig. 2 c) the sample was frozen in the setup with vertical temperature gradient by contact with liquid nitrogen, Fig. 2 b) is the cross section of the sponge along the x-axis; Fig. 2 d). the sample was frozen in the setup with vertical temperature gradient by contact with liquid nitrogen using 5\% (w/w) TBA.

Under these conditions, the nucleation and growth of air bubbles possibly increased the pressure in the sample; as a result, the ice shell breaks. Such an effect can account for macrovoid formation observed in Fig. 2 a).

The samples shown in Fig. 2 b) and Fig. 4 c) were prepared in the controlled device without TBA. The cross-sectional image of the sponge (Fig. 2 c) ) reveals the microtubule structure and parallel arrangement of the pores. the morphology of this side was plate-like when the sample was incised parallel to the x-axis (Fig. 2 b) ). This result could be explained by the planar ice crystals growing in the solution. In theory, the solid/liquid interface during freezing can be plate-like, dendriform, needle-like, and finger-like. The plate-like interface is unstable and tends to change into a complex morphology. The formation of plate-like ice crystal morphology requires the presence of a constitutional supercooled region before the planar ice front [20]. Such ice formation also requires low speed movement of the solid/liquid interface [23]. According to the research of Chalmer and Rutter [24], constitutional supercooling is induced by concentration distribution. In the present study, high $\mathrm{LCH}$ and HA concentrations increased the apparent viscosity. As water froze, solute concentration increased, producing highly concentrated solutions and becoming more viscous at lower temperatures. Thus, ice nuclei are less likely 
to form and supercooling more likely occurs. These conditions favor the formation of planar ice; as a result, a planar structure remained in the sponges after ice sublimated. TBA can also cause the breakdown of planar ice, resulting in the formation of dendritic ice [Fig. 2(d)]. The pores were unidirectional and oriented parallel to the thermal gradient. The pores varied in diameter but were partly connected. TBA decreased the supercooling effect in the system, leading to the formation of a dendritic ice structure. Some branches were connected during growth because of the anisotropic growth of ice, thereby forming the connections between pores.

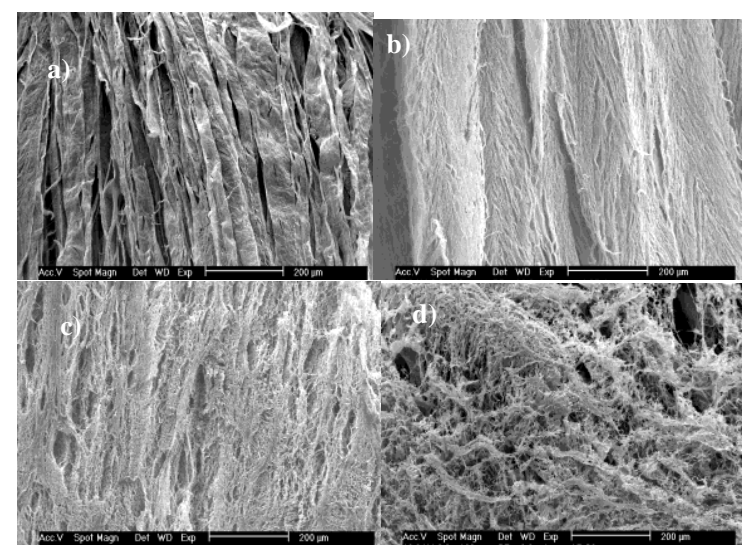

Fig. 3. Dependence of cross-section of sponge microstructure on concentration (w/w) of TBA in solution frozen in the setup with vertical temperature gradient by contact with liquid nitrogen: a). $12 \%, b) .20 \%$, c). $30 \%, \mathrm{~d}) .40 \%$.

Previous experiments were conducted using TBA and revealed that TBA can alter the crystallization behavior of ice [21], [22]. The present study also indicated that the ice morphology exhibited an arbitrary shape at higher TBA concentrations. Fig. 3 presents the morphologies of the sponges at varying amounts of TBA. In the presence of $5 \%$ TBA to $20 \%$ TBA, microtubule-like pores were formed during freezing and lyophilization. As TBA concentration was increased, the diameter of microtubules and the thickness of the microtubule walls also increased. The regularity of the microtubule pore structure was also improved. The micrographs (Fig. 3 b) ) also showed two levels of porosity at $20 \%$ TBA. Elongated, oriented microtubules with thicker walls were observed. On the other side, the surfaces of the microtubule walls consisted of many small pores arranged in parallel arrays along ridges or grooves in the surface. This two-phase structure was formed possibly as a result of the differences in ice nucleation conditions in the system. Numerous lyophilization technologies based on the use of TBA have demonstrated [22], [25] that a sufficient amount of water molecules surround TBA molecules at lower TBA concentrations; stable clathrate hydrates are then formed; water molecules bind to these hydrates, forming an ice-like cage [22]. Under these conditions, water behaves the same way as ice, which has a highly ordered structure; this behavior results in the formation of elongated ice crystals that grew parallel to the thermal gradient. However, pure ice is formed when the solution freezes, leaving an increasingly concentrated TBA solution. The amount of the remaining water molecules are insufficient to surround the TBA molecule, such that more TBA molecules can self-associate and form dimers that are linked by hydrogen bonds. The resulting low-density water clusters cannot be formed from ice-like crystals because they lack a long-range order. As pure water freezes, the concentration of the LCH-HA solution increases; this condition allows the formation of small amorphous ice grains on the microtubule walls (Fig. 3 b) ). Many smaller ice grains are formed at high concentrations; this result is consistent with that in previous studies [26], [27]. As TBA concentration increases, the morphology of amorphous ice was irregular. Numerous small pores were formed, with pleat-shaped tracings of microscopic ice formation Fig. $3 \mathrm{c}$ ) and Fig. 3 d) ).

\section{B. Permeation Properties and Water Uptake of Different Sponges}

Patients with burns lose large amounts of water through evaporation from open wounds. An ideal wound dressing would effectively protect against excessive water loss. The rate of water loss for normal skin is $204 \pm 12 \mathrm{~g} \cdot \mathrm{m}^{-2}$ per day, whereas the rate of water loss for injured skin ranges from $279 \pm 26 \mathrm{~g} \cdot \mathrm{m}^{-2}$ per day for a first degree burn to $5138 \pm 202$ $\mathrm{g} \cdot \mathrm{m}^{-2}$ per day for a granulating wound [28]. Wound dressings with very low water vapor transmission rates (WVTRs) include Vigilon. RTM., Vigilon cover film, and Stretch ' $n$ ' Seals dressings, which have WVTRs of $168 \pm 32,139 \pm 23$, and $326 \pm 44 \mathrm{~g} \cdot \mathrm{m}^{-2} \mathrm{day}^{-1}$, respectively [28]. Low WVTR results in the accumulation of exudates, which may retard healing and increase the risk of bacterial growth. By contrast, hydrophilic materials, such as Geliperm.RTM, have a high WVTR of $10972 \pm 995 \mathrm{~g} \cdot \mathrm{m}^{-2} \cdot \mathrm{day}^{-1}$. However, the use of wound dressings with high WVTRs can lead to the complete dehydration of the wound surface and the formation of eschars [29]. In addition, eschars rapidly absorb exudates, resulting in overdrying of the wound surface and may be accompanied by pain. In the present study, a linear plot of water loss versus time by the transmission area $\left(1.38 \times 10^{-3}\right.$ $\mathrm{m}^{2}$ ) was obtained. The slope of the plot was used to calculate WVTR (Fig. 4). The water vapor permeability $P$ can be obtained using Eq. (2). The moisture transmission rate of a material is referred to as its permeability, which is not dependent on the thickness of the materials. The permeance $(P / L)$ of the material, however, is dependent on thickness. Permeance is the number that should be used to compare various products in terms of moisture transmission resistance. Table I summarizes the results of the water permeability test for the sponges with different microstructures.

TABLE I: WATER VAPOUR TRANSMISSION RATES (WVTRS), Permeabilities $(P)$ AND PERmeAnce $(P / L)$ VALues of DifFERENT SPONGES

\begin{tabular}{lcccc}
\hline \hline Sample & $\begin{array}{l}\text { Thickness } \\
(\mathrm{mm})\end{array}$ & $\begin{array}{l}\text { WVTR }(\mathrm{g} \\
\left.\mathrm{m}^{-2} \mathrm{day}^{-1}\right)\end{array}$ & $\begin{array}{l}\boldsymbol{P} \times 10^{13} \\
\left(\mathrm{~kg} \mathrm{~Pa}^{-1} \mathrm{~m}^{-1}\right.\end{array}$ & $\begin{array}{l}\boldsymbol{P} / \mathbf{L} \times 10^{8} \\
\left(\mathrm{~kg} \mathrm{~Pa}^{-1} \mathrm{~m}^{-2}\right.\end{array}$ \\
$\begin{array}{l}\text { Sponge with random } \\
\text { micro-pores }^{\mathrm{a}}\end{array}$ & 4 & 452 & 70 & 17.5 \\
$\begin{array}{l}\text { Sponge with } \\
\text { microtubule-like pores }^{\mathrm{b}}\end{array}$ & 10 & 577.57 & 224 & 22.4 \\
\hline
\end{tabular}

a) Sponges were frozen in a sealed aluminum vessel by immersing in liquid nitrogen. b) Sponges were frozen in the device with a vertical temperature gradient by contacting with liquid nitrogen using $5 \%(\mathrm{w} / \mathrm{w})$ TBA as co-solvent. 
The sponges in the present experiment exhibited WVTRs ranging from $452 \mathrm{~g} \cdot \mathrm{m}^{-2} \cdot \mathrm{day}^{-1}$ for sponges with a random pore structure to $577 \mathrm{~g} \cdot \mathrm{m}^{-2} \cdot \mathrm{day}^{-1}$ for sponges with microtubule-like pores. These results suggested that the sponges are highly applicable as wound dressings. The sponges with random pores exhibited lower permeance than microtubule-like sponges. In the porous media, water transport involves three main stages: water diffusion in porous media; water diffusion in air filling the pores of the media; and water sorption/desorption at the surface of external media and internal pore surface [30]. The porous media exhibit different properties, such as size, tortuosity and type of pores, greatly influence water transport. Water molecules are found in the pore space of the membrane structure. Thus, the transport of water molecules in a hydrophilic membrane occurs by diffusion and is considered as a permeable process. For the sponges with random pores, the tortuous path reduced water permeability and could limit diffusion across the media.

Therefore, the tortuous path of the water molecules reduces the $W V T R$ of the layer. The sponges with uniform tubule-like pores provide less tortuous paths that are conducive for permeation, thereby increasing the effective path length of diffusion.

Studies on the gas permeability of wound dressing in the wet state is also significant because a $\mathrm{CH}$ membrane has a low permeability for gas in its dry state [31], [32]; such studies are also important because wound dressing should absorb exudates and maintain a wet state. For a hydrophilic polymer membrane, low permeability to gases could be further improved if this membrane in a wet state is used by water [31]. A beaker filled with saturated $\mathrm{K}_{2} \mathrm{SO}_{4}$ solution is placed at the bottom of test cell to maintain $97.5 \%$ relative humidity [33]. Fig. 5 shows an example of air flow rates for different sponges as a function of the maintained pressure gradient. We observed an ideal linear behavior in accordance with Darcy's law. The gas permeability tensor, $K$, of the sponges in the wet state could be derived from the line slope. The results shown in Table II revealed that the sponges with uniform pore microstructures exhibited considerably higher $K$ than the sponges with a random pore structure. Homogeneous open-pore structures provided low permeation resistance, which is favorable for gas molecular diffusion. The results agree with the observations in WVTR experiment.

Porosity is indirectly related to water adsorption. A porous medium with a higher porosity typically has a higher water adsorption (more open area for the flow of water) than a non-porous medium. In the present experiment, the sponges with microtubule-like pores exhibit higher porosity, resulting in higher hydraulic conductivity.

TABLE II: AIR PERMEABILITY AND SOLUTION SORPTION ABILITY OF DIFFERENT SPONGES

\begin{tabular}{lccc}
\hline \hline Sample & $\begin{array}{c}\text { permeability tensor, } \\
K, \mathrm{~m}^{2}\end{array}$ & $\begin{array}{l}\text { Porosity } \\
(\%)\end{array}$ & $\begin{array}{c}\text { Solution } \\
\text { adsorption }\left(S_{P} ; \%\right)\end{array}$ \\
$\begin{array}{l}\text { Sponge with random } \\
\text { micro-pores }^{\mathrm{a}}\end{array}$ & $1.8 \times 10^{-14}$ & 84 & 585 \\
$\begin{array}{l}\text { Sponge with } \\
\text { microtubule-like } \\
\text { pores }^{\mathrm{b}}\end{array}$ & $3 \times 10^{-12}$ & 96 & 632 \\
\hline \hline
\end{tabular}

a) Sponges were frozen in a sealed aluminum vessel by immersing in liquid nitrogen. b) Sponges were frozen in the device with a vertical temperature gradient by exposing to liquid nitrogen using $5 \%$ (w/w) TBA as a co-solvent.

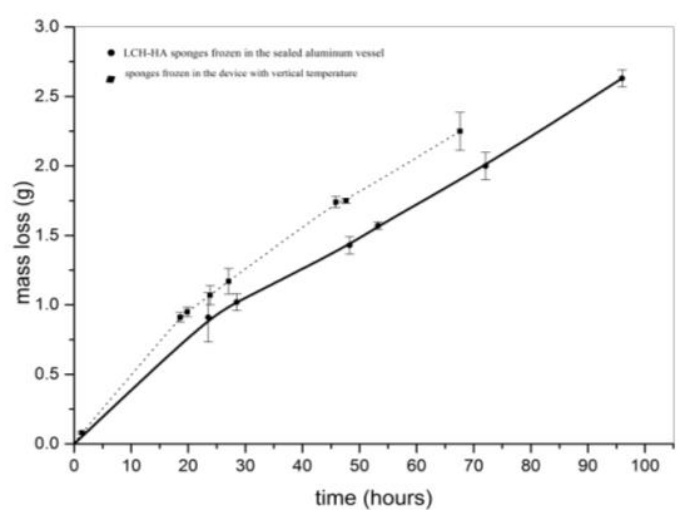

Fig. 4. Water mass loss versus time plots.

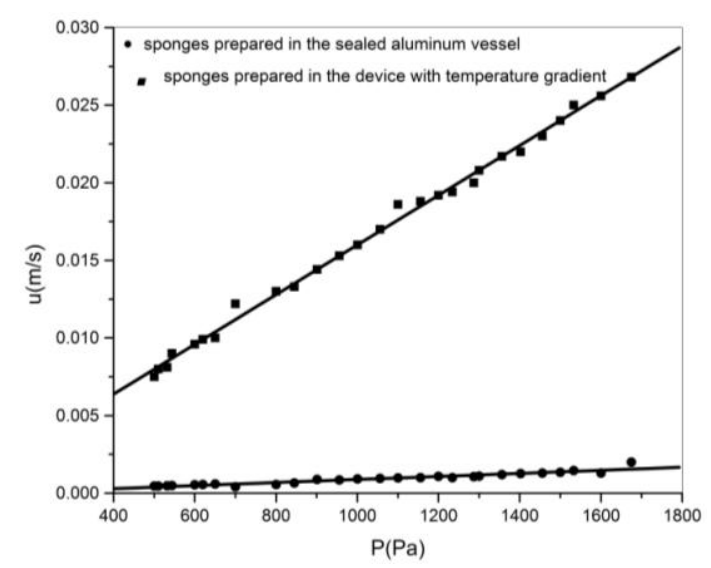

Fig. 5. The rates of air flows through the freestanding two kinds of sponge as functions of applied pressure in their wet state.

\section{In Vitro Degradation}

The sponges with random pores exhibited slightly lower degradation rates than those with tubule-like pores (Fig. 6). This characteristic may elucidate that microtubule-like pores are more permeable to water and more prone to degradation by lysozymes than random pores. Sponges with pure $\mathrm{CH}$ scaffolds degraded by lysozymes exhibit low degradation rates and can last up to several months; by contrast, the sponges prepared in the present study were degraded by approximately $80 \%$ in $5 \mathrm{~h}$. The observed phenomenon may be caused by two reasons. Firstly, water- insoluble $\mathrm{CH}$ can be modified by grafting lactic acid on amino groups without catalysis, resulting in the formation of water-soluble $\mathrm{LCH}$ [34]. In degradation, water-soluble lysozyme almost exclusively cleaves glycosidic linkages between two acetylated units [35]. Lysozyme adsorption is then enhanced by H-bond interaction and charge attraction with a hydrophilic surface [34]. Therefore, the increase in the solubility of $\mathrm{LCH}$ can accelerate the reaction rate of lysozymes and disruption of glycosidic bonds. Secondly, experiments on $\mathrm{LCH}$ have revealed that grafting by lactic acid occurs randomly along the $\mathrm{CH}$ chains in the solution, resulting in a randomly grafted $\mathrm{CH}$ and destroying the regular arrangement of the original $\mathrm{CH}$ chains [36], [32]. After lactic acid is grafted, the crystallinity of $\mathrm{CH}$ decreases and the portion of amorphous regions in $\mathrm{CH}$ increases. Degradation often occurs first in the amorphous domain. The amorphous parts are then removed and dissolved in a reaction medium during degradation, and the crystalline structure is destroyed [37]. Therefore, an increase in the amorphous domain in the sponges leads to the rapid enzymatic degradation of $\mathrm{LCH}$. 
Wound dressing with a higher biodegradation capacity than other wound dressings is non-adherent and can be removed easily without causing damage to the granulation tissue and the newly formed epithelium. Such biodegradation capacity minimizes patient discomfort when wound dressing is changed.

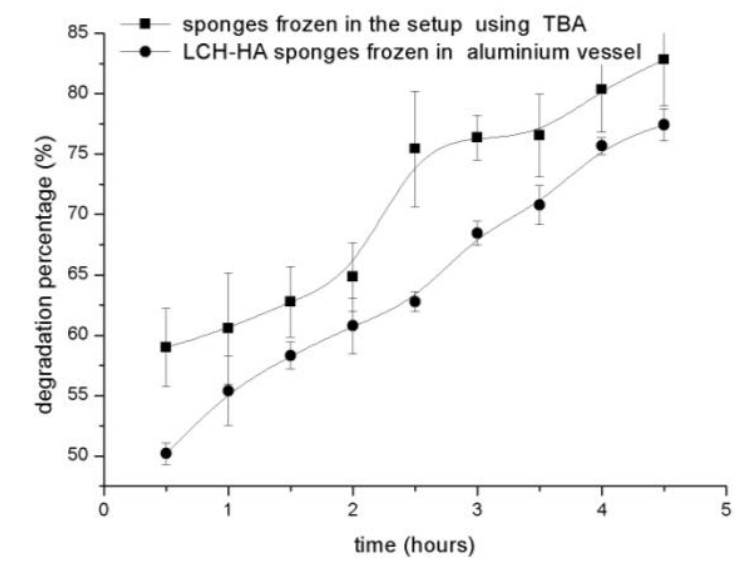

Fig. 6. Degradation percentage of different sponges by loysozyme in PBS solution.

\section{CONCLUSION}

In the present study, $\mathrm{CH}$ was modified by lactic acid without catalysis. Water-soluble $\mathrm{LCH}$ reacted with $\mathrm{HA}$ in neutral $\mathrm{pH}$, leading to the formation of sponges with high permeability properties and moisture retention abilities. LCH-HA sponges with different microstructures were prepared in the device with a vertical thermal gradient and sealed aluminum vessel.

1) For the samples prepared in an aluminum vessel without vertical temperature gradient, ice crystals nucleated homogeneously at the vessel wall. The crystals grew in all directions, forming sponges with random micropores. As the sponges were prepared in a controlled device with vertical temperature gradient, microtubule-like pores arranged in parallel were formed.

2) TBA altered the crystallization of ice, disrupting the planar ice structure; as a result, dendritic ice was formed. At lower TBA concentrations (20\%), ice exhibited a highly ordered structure, forming elongated, oriented microtubules, which grew parallel to the thermal gradient. As TBA concentration was increased, the morphology of amorphous ice was more irregular, forming small pores in the sponges.

3) The sponges in the present experiment exhibited WVTRs ranging from $452 \mathrm{~g} \cdot \mathrm{m}-2 \cdot$ day -1 for the sponges with random pores to $577 \mathrm{~g} \cdot \mathrm{m}-2 \cdot$ day -1 for the sponges with microtubule-like pores. These results suggested that the sponges with microtubule-like pores are highly applicable as wound dressing. The sponges with uniform tubule-like pores provided less tortuous path for permeation, thereby increasing the effective path length of diffusion.

4) The gas permeability of the sponges in the wet state was investigated. The sponges with uniform pore microstructures exhibited higher $\mathrm{K}$ than the sponges with random pore structures because of the low permeation resistance of uniform tubule-like pores.
5) The sponges with random pores showed slightly lower degradation rates than the sponges with tubule-like pores because the former exhibited lower accessibility to lysozyme solution than the latter. The sponges prepared in the present experiments were degraded by approximately $80 \%$ in $5 \mathrm{~h}$. This degradation rate is considerably faster than that of pure $\mathrm{CH}$ scaffolds. Lactic acid modified $\mathrm{CH}$ and decreased the crystallinity of $\mathrm{CH}$, leading to rapid lysozymatic biodegradation. The high water-solubility of LCH-HA sponges was also responsible for such rapid biodegradation.

\section{ACKNOWLEDGMENT}

This research was supported by Major State Basic Research Development Program (973 Project No.2012CB933600).

\section{REFERENCES}

[1] D. K. Singh and A. R. Ray, "Biomedical applications of chitin, chitosan, and their derivatives," J. Macromol. Sci., Rev. Macromol. Chem. Phys, vol. 40, pp. 69-83, Feb. 2000.

[2] P. H. VandeVord, H. W. T. Matthew, S. P. DeSilva, L. Mayton, B. Wu, and P. H. Wooley, "Evaluation of the biocompatibility of chitosan in mice," J. Biomed Mater Res., vol. 59, pp. 585-590, Mar. 2002.

[3] Y. L Zheng, Y. Wu, W. L. Yang, C. C. Wang, S. K. Fu, and X. Z. Shen, "Preparation, characterization, and drug release in vitro of chitosan-glycyrrhetic acid nanoparticles," J Phar. Sci., vol. 95, pp. 181-191, Jan. 2006.

[4] K. Mizuno, K. Yamamra, K. Yano, T. Osada, S. Saeki, N. Takmoto, T. Sakura, and Y. Nimura, "Effect of chitosan film containing basic fibroblast growth factor on wound healing in genetically diabetic mice," J. Biom. Mate Res, vol. 64, pp. 177-181, Jan. 2003.

[5] K. Aoi, A. Takasu, M. Okada, and T. Imae, "Nano-scale molecular shapes of water-soluble chitin derivatives having monodisperse poly (2-alkyl-2-oxazoline) side chains," Macro. Chem. Phys., vol. 200, pp. 2650- 2657, Feb. 1999.

[6] G. Kumar, P. J. Smith, and G. F. Payne, "Enzymatic grafting of a natural product onto chitosan to confer water solubility under basic conditions," Biotech Bioeng, vol. 63, pp. 154-163, Apr. 1999.

[7] S. Cafaggi, R. Leardi, B. Parody, G. Gaviglioli, E. Russo, and G. Bignardi, "Preparation and evaluation of a chitosan salt-poloxamer 407 based matrix for buccal drug delivery," J. Controlled Release, vol. 102, pp. 159-169, Jan. 2005.

[8] R. Duncan et al., "Evaluation of the biological properties of soluble chitosan and chitosan microspheres," Inter J. Phar, vol. 148, pp. 231-240, Mar. 1997.

[9] W. Weecharangsan, P. Opanasopit, T. Ngawhirunpat, T. Rojanarata, and A. Apirakaramwong, "Chitosan Lactate as a nonviral gene delivery vector in COS-1 cells," AAPS Pharm Sci Tech, vol. 7, pp. 1-6, Sep. 2006.

[10] H. H. K. Xu, J. B. Quinn, S. Takagi, and L. C. Chow, "Processing and properties of strong and non-rigid Calcium Phosphate Cement," $J$ Dental Res, vol. 81, pp. 219-224, Mar. 2002.

[11] K. Peh, T. Khan, and H. Ch'ng, "Mechanical, bioadhesive strength and biological evaluations of chitosan films for wound dressing," J Pharm Sci, vol. 3, pp. 303-311, Sep.-Dec. 2000.

[12] F. Guillaumie, B. M. Malle, K. Schwach-Abdellaoui, and T. C. Beck, "Moisturization and antiaging," Cosmetics \& Toiletries, vol. 121, pp. 51-58, Mar. 2006.

[13] L. Richert, P. Lavalle, E. Payan, X. Z. Shu, G. D. Prestwich, J. F. Stoltz, P. Schaaf, J. C. Voegel, and P. C. Icart, "Layer by layer buildup of polysaccharide films: Physical chemistry and cellular adhesion aspects," Langmuir, vol. 20, pp. 448-458, Jan. 2004.

[14] P. Kujawa, P. Moraille, J. Sanchez, A. Badia, and F. M.Winnik, "Effect of molecular weight on the exponential growth and morphology of hyaluronan/chitosan multilayers: A surface plasmon resonance spectroscopy and atomic force microscopy investigation," JACS, vol. 127, pp. 9224-9234, Jan. 2005.

[15] O. Etienne, A. Schneider, C. Taddei, L. Richert, P. Schaaf, J. C. Voegel, C. Egles, and P. C.icart, "Degradability of polysaccharides multilayer films in the oral environment:an in vitro and in vivo study," Biomacromolecules, vol. 6, pp. 726-733, Mar.-Apr. 2005. 
[16] B. Thierry, F. M. Winnik, Y. M. Erhi, and M. Tabrizian, "Nanocoatings onto arteries via layer-by-layer deposition: Toward the in vivo repair of damaged blood vessels," JACS, vol. 125, pp. 7497-7495, Jun. 2003.

[17] L. Kukolikova, D. Bakos, P. Alexy, S. Hanzelova, and W. Zhong, "Optimization of the properties of chitosan lactate/hyaluronan film," $J$ App Poly Sci, vol. 100, pp. 1413-1419, Jan. 2006.

[18] R. A. Muzzarelli, A. Isolate, and A. Ferrero, $\mathrm{CH}$ membrane, ion exchage and membranes, London: Gordon and Breach, 1974.

[19] T. Fusasawa and M. Ando, "Synthesis of porous ceramics with complex pore structure by freeze-dry processing," JACS, vol. 84, pp. 230-232, Dec. 2001

[20] H. Schoof, L. Bruns, A. Fischer, I. Heschel, and G. Rau, "Dendritic ice morphology in unidirectionally solidified collagen suspensions," J Cry Growth, vol. 209, pp. 122-129, Jan. 2000.

[21] D. J. Van Drooge, W. L. J. Hinrichs, and H. W. Fijlink, "Incorporation of lipophilic drugs in sugar glasses by. lyophilization using a mixture of water and tertiary. butyl alcohol as solvent," J Pharm Sci, vol. 93, pp. 713-725, Mar. 2004.

[22] K. Kasraian and P. P. Deluca, "The effect of tertiary butyl alcohol on the resistance of the dry product layer during primary drying," Pharm Res, vol. 12, pp. 491-495, Mar. 1995.

[23] Y. M. Xiao, H. Sun, L. Xu, H. D. Feng, and H. M. Zhu, "Thermal contact conductance between solid interfaces under low temperature and vacuum," Rev Sci Instrum, vol. 75, pp. 3074-3076, Sep. 2004.

[24] J. W. Rutter and B. Chalmer, "A prismatic substructure formed during solidification of metals," Can. J. Phys, vol. 31, pp. 15-39, Jan. 1953.

[25] M. C. R. Symons and M. J. Blandamer, In hydrogen-bonded solvent systems (E.D.S Covington and Jones), London, 1968.

[26] V. S. Madihally and H. W. T.Matthew, "Porous chitosan scaffolds for tissue engineering," Biomaterials, vol. 20, pp. 1133-1142, Jun. 1999.

[27] J. S. Mao, L. G. Zhao, Y. J. Yin, and K. D. Yao, "Structure and Properties of Bilayer Chitosan-gelatin Scaffolds," Biomaterials, vol. 24, pp. 1067-1074, Mar. 2003.

[28] Y. D. Sanzgiri, L. M. Benedetti et al., "Structure and properties of bilayer chitosan-gelatin scaffolds," Biomaterials, vol. 17, pp. 1639-1643, Mar. 1996.

[29] M. Kokabi, M. Sirousazar, and Z. M. Hassan, "PVA-clay nanocomposite hydrogels for wound dressing," Euro Poly J, vol. 43, pp 773-781, Mar. 2007.

[30] H. Schmidt, D. Marcinkowska, and M. Cieslak, "Testing water vapour permeability through. porous membranes," Fibres \& Textiles in Eastern Europe, vol. 13, pp. 66-69, April / June 2005.

[31] A. Ito, M. Sato, and T. Anma, "Permeability of $\mathrm{CO}_{2}$ through chitosan membrane swollen by water vapor in feed gas," Die Angewandte Makromolekulare Chemie, vol. 24, pp. 85-94, Mar. 1997.
[32] D. Depan, A. P. Kumar, and R. P. Singh, "Preparation and characterization of novel hybrid of chitosan-g-lactic acid and montmorillonite," J Biom Mater Res A, vol. 78A, pp. 372-378, Aug. 2006.

[33] P. W. Winston and D. H. Bates, "Saturated solutions for the control of humidity in biological research," Ecology, vol. 41, pp. 232-237, Mar. 1960.

[34] M. N. V. Ravi Kumar, R. A. A. Muzzarelli, C. Muzzarelli, H. Sashiwa, and A. J. Domb, "Chitosan chemistry and pharmaceutical perspectives," Chem Rev, vol. 104, pp. 6017-6084, Dec. 2004

[35] S. K. Kim and N. Rajapakse, "Enzymatic production and biological activities of chitosan oligosaccharides (COS): A review," Carbon Polym, vol. 62, pp. 357-368, Dec. 2005.

[36] X. Qu, A. Wirsen, and A. C. Albertsson, "PH-sensitive hydrogels based on chitosan and D, L-lactic acid," ACS Polym Mater Sci Eng, vol. 79 , pp. 242-243, Dec. 1998.

[37] F. L. Mi, S. S. Shyu, Y. B. Wu, S. T. Lee, J. Y. Shyong, and R. N. Huang, "Fabrication and characterization of a sponge-like asymmetric chitosan membrane as a wound dressing, Biomaterials," vol. 11, pp. 165-173, Feb. 2001

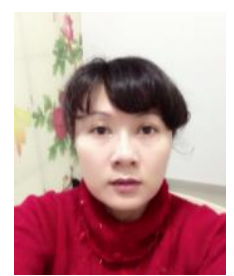

Chen Lai was born in People's Republic of China at tenth November. She got her bachelor degree in chemical engineering at College of Chemical Engineering, Beijing University of Chemical Technology, China in 1996. In 2002, she got her master degree in materials science in the College of materials science and technology, Hunan University (NUT), China. From June 2003 to June 2006, she was a guest student at Key Laboratory of Specially Functional Materials and Advanced Manufacturing Technology of Ministry Education, College of Materials Science and Engineering, South China University Of Technology (SCUT), China. She got her Ph.D. degree in materials science in the college of materials science and technology, Hunan University (NUT), China in 2006.

Her work experience including: being a Water Quality analyst from July 1996 to July 1999 in the Power Plant, Balin Petro-Chemical CO., LTD, China Petrochemical Corporation (sinopec)being a postdoctor of biomaterials from June 2006 to December 2008 in the Key Laboratory of Specially Functional Materials and Advanced Manufacturing Technology of Ministry Education, College of Materials Science and Engineering, South China University of Technology (SCUT), China.

Her current and previous research concerns bacterial cellulose based biomaterials as the wound dressing and bone repairing. 